\title{
La educación geográfica contemporánea, la geografía escolar tradicional y el pensamiento crítico
}

\section{Contemporary geographic education, traditional school geography and critical thinking}

\author{
José Armando Santiago Rivera \\ Profesor. Universidad de Los Andes, Venezuela. \\ asantia@ula.ve, jasantiar@yahoo.com | 0000-0002-2355-0238
}

\begin{abstract}
Para citar este artículo: Santiago Rivera, J. A. (2021). La Educación geográfica contemporánea, la geografía escolar tradicional y el pensamiento crítico. Entorno Geográfico, (21), 157-179.

https://doi.org/10.25100/eg.v0i21.11297
\end{abstract}

\section{Resumen}

El propósito es reflexionar sobre la educación geográfica contemporánea, la geografía escolar tradicional y el pensamiento crítico. Se trata de tres aspectos que facilitan entender las razones de mejorar su calidad formativa, en las condiciones del mundo contemporáneo. Eso supone explicar los fundamentos de la Educación Geográfica, el afecto a la transmisión de contenidos decimonónicos y el incentivo del pensamiento crítico. Metodológicamente se realizó una revisión documental para plantear el análisis sobre la situación de la Educación Geográfica contemporánea, la problemática característica de la geografía escolar tradicional y la necesidad de promover el pensamiento crítico como opción para superar el afecto a la memorización. Se concluye al subrayar la necesidad de superar las contradicciones derivadas de esa relación pedagógica, como impedimento que obstaculiza comprender la complejidad ambiental, geográfica y social globalizada y sus efectos en la calidad de vida de la colectividad mundial.

Palabras clave: Educación Geográfica Contemporánea, Geografía Escolar, Pensamiento crítico.

\begin{abstract}
The purpose is to reflect on contemporary geographic education, traditional school geography, and critical thinking. These are three aspects that facilitate understanding the reasons for improving their educational quality, in the conditions of the contemporary world. This means explaining the fundamentals of Geographical Education, the affection for the transmission of nineteenth-century content and the incentive for critical thinking. Methodologically, a documentary review was carried out to propose the analysis on the situation of contemporary


Geographical Education, the characteristic problems of traditional school geography and the need to promote critical thinking as an option to overcome the affect on memorization. It concludes by underlining the need to overcome the contradictions derived from this pedagogical relationship, as an impediment that hinders understanding the globalized environmental, geographical and social complexity and its effects on the quality of life of the world community.

Keywords: Contemporary Geographical Education, School Geography, Critical thinking.

Recibido: 17 de diciembre de 2020

Aceptado: 02 de febrero de 2021

\section{Introducción}

La situación geográfica característica del inicio del nuevo milenio revela en el mundo contemporáneo, un comportamiento contradictorio. Mientras hay un extraordinario desarrollo científico y tecnológico, se visibiliza una realidad colmada de necesidades, dificultades y contratiempos, en su mayoría, originados por la forma cómo se relacionan los grupos humanos con sus territorios. Esa preocupante realidad emerge de la práctica realizada por el capital financiero al intervenir la naturaleza y propiciar las condiciones causantes de la ruptura del equilibrio ecológico globalizado.

La demostración de esa relación sociedad y naturaleza tiene como perfil evidente, los casos revelados en la diversidad de eventos socioambientales y geográficos de suceder cotidiano en las diversas regiones del mundo globalizado, por ejemplo, la disminución de los glaciares, en Europa, Asia y América; el desequilibrio de los pisos biogeográficos, las lluvias copiosas y las inundaciones; el deshielo de los glaciares, tanto en el Ártico como en la Antártida. A muchos de estos casos, se les asignan como causalidad el calentamiento global, el cambio climático y/o la contaminación ambiental.

El conocimiento actual sobre esta situación apunta a resaltar el acontecer de sucesos con rasgos destructivos y devastadores, considerados como temas de interés colectivo para la comunidad mundial. Al respecto, se fomentan eventos académicos, cuyo propósito es compartir conocimientos y prácticas sobre la formulación de opciones factibles de reconstruir el sistema ecológico integral del planeta, disminuir los efectos de calentamiento global y potenciar 
alternativas factibles de restituir afanosamente el cambio climático, ante el reto de fundamentar el desarrollo sustentable hacia una naturaleza humanizada.

La importancia asignada por los expertos que analizan esta situación, desde fines del siglo XX hasta el presente momento histórico, han demostrado el afán por lograr con efectividad, una versión de la relación sociedad y la naturaleza, desde una perspectiva sensibilizadora racional y reconstructiva sobre lo finito de las condiciones del planeta. De esta manera la urgencia es promover una acción interventora lo menos destructiva posible y preservar recursos naturales para las futuras generaciones. De allí el interés en facilitar una educación más centrada en promover una innovada conciencia ambiental y geográfica.

Sin embargo, cuando se reflexiona sobre la exigencia que la geografía escolar asuma la disposición de contribuir a mejorar la complicada problemática ambiental de alcance mundial, los expertos cuestionan la permanencia de los fundamentados geográficos, pedagógicos y didácticos de origen en el siglo XIX. Es la estabilidad del modelo educativo transmisivo, libresco, reproductor, memorístico y circunscrito al aula de clase, con una muy escasa relación con la explicación del entorno inmediato. Esta tarea causa una educación intelectualizadora preocupada tan solo por facilitar contenidos programáticos absolutos.

Esto supone considerar como dificultad formativa, el desarrollo de un acto educante circunscrito a la escuela y notablemente descontextualizado del deterioro ecológico, ambiental y geográfico local. Esa actividad informativa fomenta el entendimiento de lo real con conductas relacionadas con la pasividad, la neutralidad, la desideologización y el apoliticismo. Esa circunstancia exige opciones formativas con la factibilidad de innovar el acento formativo con el fomento del pensamiento crítico, como la base de la conciencia cuestionadora y constructiva ante el deterioro destructivo de las condiciones naturales.

Metodológicamente este objeto de estudio determinó realizar una revisión bibliográfica y estructurar un planteamiento que reflexiona sobre la Educación Geográfica contemporánea, la geografía escolar y el pensamiento crítico. En este caso, la situación de la Educación Geográfica como el contexto de la explicación; luego reflexiona sobre la Educación Geográfica y la 
geografía escolar y, a continuación, asume una postura analítica sobre la Educación Geográfica, la geografía escolar y el fomento del pensamiento crítico.

\section{La situación de la Educación Geográfica}

A fines del siglo XX, en los medios de comunicación se reveló la preocupación con alcance planetario sobre el convencimiento de la existencia de los problemas calificados como Desastres Naturales, con el signo de catástrofes, calamidades, desgracias y adversidades. Así, en las realidades divulgadas por la acción mediática, fue común asignar la causalidad al hecho como si fueran respuestas facilitadas por la naturaleza y denunciar el tratamiento social de los territorios. En efecto, la culpabilidad tuvo el desacierto de centrarse en la naturaleza y desvió la atención sobre la injerencia de capital.

Esta contradicción significó obviar la acción económico-financiera, cuyo interés ha sido incrementar la capacidad del beneficio factible de alcanzarse con el apoyo de las novedades tecnológicas y obtener una excesiva rentabilidad financiera en el aprovechamiento de los recursos de los territorios. El hecho permitió invisibilizar al capital y sus propósitos, dados sus efectos en el inocultable deterioro ocasionado por la labor destructiva de las corporaciones multinacionales, conformadas por el capitalismo hegemónico globalizado, con el propósito de acumular riqueza, con la destrucción ambiental, evidente en los altos niveles del deterioro ecológico (Ander-Egg, 2004).

Sin embargo, la magnitud de las dificultades se hizo cada vez más preocupante, porque fue imposible ocultar sus nefastas consecuencias. En principio, la atención se centró en problemas de mayor escala geográfica, en cuanto a su alcance. Al respecto, se pueden considerar ejemplos de esa situación, la acumulación de basura en el centro del océano pacífico, el aumento del nivel del mar, el deshielo del casquete polar del norte, el quiebre de la estabilidad de las estaciones, tanto al norte como en el sur; la contaminación ambiental en las ciudades y el hacinamiento urbano.

Asimismo, a escala de las comunidades, los efectos nefastos de la contaminación ambiental, se mostraron con un desenvolvimiento inquietante, en lo referido a la acumulación de la basura, la contaminación sónica, la construcción de viviendas en áreas poco propicias por sus inestables condiciones territoriales, la falta de planificación urbana, la contaminación del aire, la deforestación, entre otros casos. Así, el deterioro ecológico y ambiental urbano mostró un 
escenario geográfico hostil, adverso, desfavorable y precario para debilitar las condiciones de la habitabilidad social (Santos, 2004).

La ruptura del equilibrio natural amerita de otras opciones epistémicas, con la capacidad de echar las bases de una comprensión del mundo y de las situaciones complejas ocasionadas por el deterioro de la naturaleza. Es de urgencia vislumbrar alternativas, cuyos propósitos apunten en la dirección de educar cualidades para comprender las razones de las inquietantes circunstancias territoriales, tanto en las localidades, como en las diversas regiones del mundo. Por tanto, eso justifica entender la realidad geográfica con un acto educante confrontador de las condiciones naturales comunitarias y la facilitación de actividades pedagógicas para fomentar el debate constructivo (Aiello, 2005).

Es colocar en el primer plano a la educación geográfica, concebida como la acción formativa facilitada a los ciudadanos con la capacidad de conocer, analizar, explicar y transformar su ambiente inmediato, con fines de mejorar su calidad de vida en sano equilibrio con las condiciones naturales del territorio habitado. Es educar para comprender la compleja situación derivada de la forma como los grupos humanos aprovechan las condiciones de su territorio y construyen su contexto geográfico, al utilizar el nivel del desarrollo científico y tecnológico alcanzado históricamente (Santiago, 2013).

En lo especial, es el acto educativo con la posibilidad de formar los ciudadanos con la capacidad pedagógica de interpretar las condiciones geográficas de su comunidad. Necesariamente eso implica cumplir con un acto educativo más coherente y pertinente con el cambio a las complicadas realidades geográficas que vive la sociedad contemporánea. En principio, es contribuir con una nueva lectura de las circunstancias ecológicas, ambientales, geográficas y sociales. Eso debe significar igualmente el fomento del pensamiento crítico y constructivo que facilite el ejercicio de la reflexión analítico-interpretativa sobre los acontecimientos-problema. Un punto de partida es asumir lo siguiente:

La geografía tiene la virtud de ser una ciencia popularizada como disciplina escolar. Tal virtud, no obstante, causa muchos de sus vicios. El "filtro" de un discurso científico que debía hacerse comprensible a las gentes exigió la "esterilización" de toda alusión que pudiera calificarse de politizada, buscándose en la escuela una 
geografía "neutra". La consecuencia es que ésta llegó a ser definida como una ciencia descriptiva, incapaz de satisfacer muchas de las exigencias aquí anotadas. La geografía tradicionalmente aprendida en las escuelas estaba ligada al sistema educativo de una época. (Soares y Ueda, 2002, pp. 90-91).

Con este perfil curricular la geografía en la escuela se comporta en la dirección contraria a la aspiración de explicar críticamente la realidad, con el incentivo de la participación activa y protagónica de los ciudadanos, cuya capacidad interpretativa, favorezca comprender el mundo vivido de manera racional, lógica y reflexiva; es decir, promover el salto epistémico desde la contemplación de lo real, con fines de convertirse en actores especuladores pasivos, imparciales y neutrales, concebidos como destinatarios de informaciones superficiales e insustanciales.

Aunque esta enseñanza geográfica ha sido cuestionada reiterativamente por su permanencia en la escuela, es inevitable reconocer que todavía subsiste con extraordinaria certeza, para traer contratiempos a su labor entendida con el calificativo de la geografía escolar. La permanencia obedece a su estabilidad, inmutabilidad y firmeza, pues ha resistido desde fines del siglo XVIII, hasta el momento contemporáneo, a las innovaciones realizadas, en principio, en los planes de estudio y, recientemente, con las frecuentes reformas curriculares para modernizar su labor pedagógica (Gallego y Pérez, 2003).

Así, la Educación Geográfica desde el siglo XIX hasta el presente, se caracteriza por ser inadecuada, impropia, inapropiada y contraproducente con las realidades geohistóricas contemporáneas. Allí, la prioridad asignada a los fundamentos de la modernidad, tiene como consecuencia, vigorizar el retardo y el aplazamiento, discrepante de la relatividad en lo teórico y lo metodológico. Eso impide la formación responsable y comprometida de la conciencia crítica y constructiva, con la disponibilidad de asegurar una propuesta educativa factible de contribuir a la innovación de formación geográfica (Maldonado, 2016).

Ante la vigencia de lo moderno, hoy día la manifestación hegemónica de la acción política ideologizadora del capital, expresada en el mercado único mundial y en el pensamiento único, se impone promover la alfabetización geográfica sustentada en el ejercicio de la dialéctica y la crítica constructiva. En efecto, se puede asegurar los claros fines del capitalismo para promover invisibilización de los desafíos del cambio socio-histórico, impedir la conciencia liberadora de los 
pueblos pobres y preservar su ignorancia y atraso para preservar el contexto del subdesarrollo y la dependencia (Balaguer, 2018).

En el propósito de su renovación, la orientación educativa de la geografía ha pretendido promover la formación integral de los ciudadanos, en lo referido a lo sano, culto y crítico, en estrecha relación y coherencia con las condiciones de la época contemporánea. Sin embargo, resulta notablemente evidente e inocultable, la persistencia de los fundamentos decimonónicos. Lo cierto es la continuidad de la transmisión de contenidos programáticos relacionados con las características físico-naturales de los territorios. Así, se vigorizan los efectos de su rezago en la escuela, aunque tan solo resulta importante en los programas radiales y televisivos, al describir y narrar aspectos de cultura general.

También, la geografía escolar como la base de la Educación Geográfica, manifiesta la discrepancia originada con su desfase del momento histórico y, en especial, de los acontecimientos comunitarios. Por tanto, en abrigarse en el enciclopedismo, el énfasis libresco y en la memorización. Con esta situación, implica concebir la enseñanza geográfica como labor formativa afecta al tradicionalismo que opaca la gestión formativa para educar ciudadanos analíticos, críticos y creativos. De esta forma, se obstaculiza el incentivo de fortalecer la subjetividad analítica, crítica y creativa, al descartar la memoria comprensiva y construir puntos de vista coherentes y argumentados (Salgado, 2011).

Lo anterior supone la salvedad y la exclusión del desarrollo del pensamiento crítico, al preservar la memorización. Eso trae como consecuencia problematizar la realidad e intervenir con una acción pedagógica orientada a valorizar la experiencia ciudadana, en la explicación de los problemas de la vida real, con fines de descifrar las razones explicativas su causalidad, desenvolvimiento y transformación. El propósito es ejercitar las reflexiones analítico-críticas y entender el momento histórico, en sus temáticas y problemáticas, como de sus repercusiones político-sociales e ideológicas.

Dentro de esta perspectiva, la Educación Geográfica debe relacionar sus propuestas de transformación en la formación de los ciudadanos en el inicio del nuevo milenio, la dinámica que 
se ha impuesto en el ámbito económico, ahora más nutrido de los avances tecnológicos y con eso, la innovación de los procesos productivos (Casas y Botella, 2003).

Igualmente, en el ámbito social, se ha impuesto la integración fomentada por los movimientos migratorios en diversas regiones del mundo. Este acontecimiento es revelador de la coexistencia multicultural o pluricultural, desenvuelta en un ámbito dinamizado por la diversidad de lenguas, religiones y expresiones culturales (Casas y Botella, 2003).

Además, la sociedad mundial está integrada en una comunidad intensamente comunicada, gracias al apoyo tecnológico de la ingeniería de la microelectrónica que ha creado y potenciado los métodos, las técnicas, los procedimientos y los recursos con la capacidad para fomentar la industria satelital. El resultado de esa tarea es la innovación de los medios de comunicación social y la posibilidad de los ciudadanos dispersos en las comunidades que conforman la unidad mundial, de estar informados al instante y en forma simultánea, ante la posibilidad de recibir las noticas sobre los acontecimientos relacionados con los numerosos eventos ocurridos en la diversidad geográfica mundial.

El acercamiento planetario ha originado para la acción mediática la oportunidad para ofrecer planteamientos con el alcance planetario, pero igualmente consolidar puntos de vista alienantes y manipuladores hacia el logro del pensamiento único. Significa que los medios se confabulan con informaciones preparadas para desvirtuar las concepciones de los ciudadanos sobre determinado tema y conformar una opinión carente de confiablidad, aunque básica en su propósito de construir una explicación somera y convincente sobre la realidad de temas relacionados con una opinión pública sesgada. (García Martínez, 2007).

La intención es crear una perspectiva que logre desnaturalizar la verdad cierta y demostrable, por una apreciación donde subyace el acento discordante, pero con una muy próxima veracidad, cuyo sentido comunicacional implica lo cierto, lo innegable, lo irrefutable y lo indiscutible. Lo subyacente representa potenciar una autenticidad maquillada con fines de desaviar la intencionalidad explicativa. Indiscutiblemente esta circunstancia es común y natural, tanto en los medios, en especial, la televisión, al igual que en las redes sociales. El objetivo es imponer una perspectiva con efectos deshonestos e inmorales. 
Al crear espacios televisivos con fines de cautivar la audiencia, se tiene como propósito internalizar comportamientos amenazantes e intimidantes en personas incautas e ingenuas. Es la captura de la conducta individual inocente que, controladas y conducidas, asumen la admiración como respuesta a la exposición cautivadora de escenas atractivas, pero preparadas con la finalidad de alejar al espectador del entendimiento de las razones que las explica. Aunque con la neutralidad, la pasividad y la desideologización, se pretende fortalecer la indiferencia ante las calamidades geográficas (Davini, 2015, p. 13).

Necesariamente, esto influye en forma decisiva en crear razonamientos plenos de superficialidad, trivialidad e impertinencia. Allí, priva la ligereza y, con eso, la generación de una verdad, cuyos motivos son la alienación, la paranoia, la fantasía y la alucinación. Estos efectos son condicionantes que impiden el fomento de la reflexión analítica e interpretativa y dar paso a la especulación. Lo preocupante es distorsionar la verdad real, objetiva y concreta, por ejemplo, en la realidad social, cuyos temas y problemas deben ser tratados con la certeza garante de la sinceridad, lo auténtico y lo veraz.

En el caso de la enseñanza de la geografía se promueve para sustentar una educación geográfica coherente con las realidades del mundo contemporáneo, la necesidad de construir una verdad distinta, diferente y confiable, por ejemplo, la memorización, resulta notablemente afectada por la multiplicidad de las noticias, informaciones y conocimientos (García Martínez, 2007). Igualmente, se coloca en el escenario de la controversia a la verdad mediática. El aspecto impulsador del debate y la polémica, es superar el pensamiento único por la promoción del pensamiento reflexivo, crítico y constructivo.

Por otro lado, esta renovación también implicó una modificación de la didáctica específica de la disciplina, por cuanto aquella geografía de antaño, en sus rasgos generales, tenía su correlato en prácticas docentes tradicionales y conductistas, donde los alumnos revestían un lugar de meros receptores pasivos. En la perspectiva renovadora, se promueven selecciones y tratamientos de esos contenidos desde una óptica de la didáctica motorizada por la pregunta, la hipótesis, la reflexión y la utilización de estrategias didácticas innovadoras. (García Ríos, 2019, pp. 181). 
De allí la importancia asignada a facilitar los procesos de enseñanza y de aprendizaje en la geografía escolar, con la investigación de los problemas ambientales, geográficos y sociales de la comunidad local. Desde esta perspectiva, el estudio del entorno cercano a la escuela, se asume como el ámbito real, inmediato y el escenario habitado, como la posibilidad de explicar el lugar habitado. Indiscutiblemente analizar lo próximo facilita formar una concepción personal, desarrollada con aprendizajes significativos y forjadores de la conciencia crítica, luego de interpretar su espacio geográfico con el testimonio de los ciudadanos. Con eso, reivindicar la subjetividad colectiva.

\section{La Educación Geográfica y la geografía escolar}

La situación enunciada de la Educación Geográfica es motivo suficiente para demandar un modelo educativo más allá de la transmisión de contenidos programáticos y la exigencia de la memorización como la manifestación del aprendizaje. Es obligatorio asumir una formación, cuya labor pedagógica contribuya a educar en forma coherente a los ciudadanos, y en la que se promueva la comprensión analítica, critica y constructiva de las condiciones sociohistóricas de la compleja época actual. Es imprescindible entender lo que ocurre, en especial, concientizar sobre el deterioro ambiental y geográfico en el ámbito de la democracia y la paz (Casas y Botella, 2003).

El desafío obedece a la exigencia de realizar la explicación a las diversas, difíciles y disimiles problemáticas percibidas en las numerosas regiones del mundo globalizado, cuyo desenvolvimiento requiere de análisis holográmicos, holísticos, integrales, además de una perspectiva de totalidad. Eso obedece a la coexistencia de las dificultades tradicionales, como los problemas de nueva factura, cuya presencia incrementan las condiciones de complejidad, confusión y preocupación de la sociedad, ante la aparente concordia de visibles e invisibles conflictos. A eso se asocia la impresionante mutabilidad en la dinámica histórica asociada a la ausencia del compromiso y la responsabilidad social por un mundo mejor (Maldonado, 2016).

Esta situación ha significado para la Educación Geográfica el apremio de atender los retos que caracterizan las realidades geográficas del mundo contemporáneo. Al respecto, se imponen respuestas convincentes relacionadas con conocimientos y prácticas pertinentes para desarrollar los procesos de enseñanza y de aprendizaje hacia la explicación interpretativa de la realidad geográfica. En principio, enfatizar en la discusión sobre el estudio de aspectos relacionados con 
la disciplina o promover la investigación de los problemas geográficos. Igualmente, preservar las actividades didácticas tradicionales o motivar el desarrollo del estudio de las problemáticas comunitarias (Aiello, 2005).

Adicionalmente, preponderar la transmisión de contenidos programáticos o facilitar las explicaciones a partir del análisis investigativo de lo real; otro aspecto a destacar lo representa el hecho de fundamentar la Educación Geográfica con los conocimientos y prácticas de la concepción científica positivista, cuando mientras tanto, en las ciencias sociales se facilitan las investigaciones con los fundamentos del enfoque cualitativo. De esta forma, se acentúa la distancia entre la finalidad educativa de formar los ciudadanos para entender críticamente la realidad geográfica vivida, al permanecer la transmisión de conocimientos y formar ciudadanos intelectualizados (Casado y Calonge, 2001).

Lo ideal debería ser afrontar las circunstancias construidas por los grupos humanos y entender las razones de su existencia, en sus temáticas y problemáticas, como las implicaciones de lo ideológico y lo político en lo real. En este caso, lo conveniente sería promocionar la actividad reconstructiva para comprender las diversas formas de apreciar el mundo, la realidad y la vida, con la acción transformadora impuesta por quienes ejercen el control hegemónico desde el capital. De allí la demanda de reivindicar en el ámbito educativo, una Educación Geográfica que permita identificar, mejorar y transformar los problemas de la sociedad y humanizar el territorio (García-Lastra, 2013).

En este marco epistémico se debe comprender la relación existente entre la Educación Geográfica y la geografía escolar. El motivo es que mientras la Educación Geografía establece las directrices para guiar el desarrollo curricular de la geografía escolar hacia una formación humanizadora, la labor pedagógica de la práctica escolar cotidiana, se desarrolla con una marcada diferencia entre lo teórico establecido en la finalidad educativa y el desempeño en el aula de clase; especialmente, en lo referido a su notable distancia de la realidad inmediata. Eso supone que, a pesar de las reiteradas propuestas de acento innovador, en lo referido a las teorías geográficas, pedagógicas y didácticas, en la geografía en la escuela, permanecen en condiciones de actualidad, los procesos de enseñanza y de aprendizaje sugeridos en el siglo XIX. 
Por ejemplo, el mundo contemporáneo se revela como escenario donde coexisten dificultades ambientales, geográficas y sociales de diversa índole. Se trata de una situación complicada debido a la contradicción entre el desarrollo científico y tecnológico, con la problemática social de graves efectos en las distintas regiones del mundo globalizado. Tal es el caso del contraste entre la opulencia y la pobreza. Desde este punto de vista, no hay país afectado por la relación entre la acumulación de capital y la miseria. Aunque eso es una ocurrencia común y natural de la época contemporánea, la geografía escolar define su propósito formativo en describir los aspectos comunes en los territorios (Santiago, 2017).

Por eso es razonable considerar que desde la Educación Geográfica se debe promover la tarea formativa de la geografía escolar con el fomento de otras versiones pedagógicas y didácticas que tengan efectos innovadores y más allá de las explicaciones científicas positivistas; por ejemplo, con los fundamentos paradigmáticos de la orientación cualitativa de la ciencia. En esa dirección, en forma prioritaria, la geografía escolar debe considerar mermar la influencia geográfica pedagógica descriptiva, al aferrarse a transmitir contenidos geográficos y descartar la memorización como la manifestación del aprendizaje. También es imprescindible que la docencia en las asignaturas geográficas, debe ser facilitada por educadores con formación disciplinar y pedagógica $\mathrm{y}$, es importante apreciar que los contenidos geográficos se diluyen en el ámbito curricular de las Ciencia Sociales.

Por tanto, se requiere que, en los procesos de la enseñanza y el aprendizaje, se promuevan cambios formativos, a la vez que se estimule el tratamiento de temas, tales como la protección del ambiente y aprovechar el territorio en forma racional, analítico y reflexiva, al igual estructurar el comportamiento social del espacio y educar los ciudadanos con capacidad para mejorar su calidad de vida. El cambio debe avanzar desde la memorización de los rasgos del territorio por la interpretación, a partir de la subjetividad de quienes lo conocen.

La educación geográfica permite desarrollar habilidades analíticas de la realidad espacial a partir del estudio de las dinámicas y contradicciones que ella contiene en su constante relación con los grupos humanos, posibilitando así, una comprensión crítica del mundo. Sin embargo, la riqueza que envuelve la geografía escolar se difumina en la cotidianeidad de las escuelas donde predomina una visión estática del espacio geográfico y prácticas pedagógicas sustentadas en el tradicionalismo..., un sistema escolar que impide el ingreso de nuevas perspectivas y no da tiempo para la 
actualización docente, incapacidad didáctica de ingresar temas y desarrollar habilidades complejas como son las relacionadas al pensamiento crítico social, lo cierto es que siendo todas o sólo alguna de ellas la principal razón para explicar la realidad de la educación geografía escolar actual, ésta en su dimensión práctica, no ha logrado renovarse en conjunto con su par disciplinar y no da muestras de permitir el ingreso a las aulas de una geografía crítica. (Salgado, 2011, p. 4).

Es un salto para colocar en tela de juicio la tradicional versión pedagógica de la geografía escolar que orienta la enseñanza, desde la versión del positivismo geográfico, al pretender realizar la comprensión de los territorios, a partir de un encadenamiento de sus caracteres físico-naturales. Se trata de una simplificación demostrada con la enumeración de los aspectos que conforman la expresión de lo natural, desde un planteamiento determinista. Al respecto, se enuncian los detalles definidores del territorio y se detallan los aspectos referidos a la cultura del lugar, en sus costumbres y tradiciones.

Con esta perspectiva, la geografía escolar marca distancia significativa de lo relacionado con la dinámica demostrativa de la presencia social y con eso, dejar a un lado la explicación de los cambios y transformaciones generadas por la colectividad comunitaria, al aprovechar sus potencialidades territoriales. Así, se privilegia la concepción geográfica tradicional. Es entonces una enseñanza limitada a detallar el territorio y descarta la presencia activa y protagónica de los ciudadanos como sus habitantes. De esta forma, enseñar significa conducir el proceso formativo desde los planteamientos establecidos por Ratzel en su propuesta geográfica y descuidar la perspectiva posibilista de Paul Vidal de la Blache. (Pipkin, Varela y Zenobi, 2001).

Quiere decir que en la enseñanza de la geografía escolar se revela la ausencia de los fundamentos geográficos formulados en la disciplina desde mediados del siglo XX. Tal es el caso de la geografía explicativa, la nueva geografía, la geografía de la percepción, la geografía radical, la geografía humanística y la geografía cultural. En este sentido, en la geografía en la escuela evidentemente se percibe el apego a la versión disciplinar tradicional como la exclusiva orientación para tan solo describir un territorio idealizado, abstracto y neutro; es decir, se destaca la acción meramente descriptiva y narrativa, cuyo propósito es observar, describir, copiar, calcar y dibujar. 
Un cuestionamiento reiterativo lo constituye de dejar a un lado la acción pedagógica y didáctica conducente a aplicar el análisis curioso y examinador de las razones explicativas del objeto de estudio. Esto representa para la Educación Geográfica de orientación positivista, desnaturalizar la acción interventora irracional de la naturaleza, cuyo propósito es ejercer el control de la economía mundial. Por tanto, su presencia en la escuela, se ha circunscrito a promover los procesos de enseñanza y de aprendizaje, con un énfasis eminentemente informativo, instrumental e instruccional y educar ciudadanos neutrales ideológicamente, apolíticos y enfáticamente pasivos en su comportamiento social (Pérez Jiménez, 2003).

En un momento donde se exige la democracia y la paz, como el desafío de la formación educativa, enseñar geografía escolar, en su práctica pedagógica cotidiana, se promueve una educación sustraída de la política estimuladora de la acción participativa y protagónica. Por esta razón, se ofrece un conocimiento exiguo, insignificante e intrascendente, además de inofensivo, insustancial e inútil. En efecto, notablemente contradictoria con la geografía calificada de subversiva, insubordinada e inconveniente. En otras palabras, por el hecho de tener la posibilidad de desnudar la ideología y la acción política para ordenar el territorio y visibilizar las contradicciones espaciales entre el capital y la pobreza.

Además, la Educación Geográfica resulta afectada por una penosa situación que se manifiesta en forma común en el escenario escolar. Se trata del rechazo al cambio en la aplicación innovadora de los avances disciplinares, pedagógicos y didácticos. Este aspecto merma las posibilidades de ofrecer una acción renovada para promover una formación educativa acorde con las condiciones históricas de la época contemporánea. Evitar el cambio de la labor cotidiana del aula de clase, obedece a la escasamente significativo de cumplir la tarea con una actividad centrada en el dictado, la copia, el dibujo y el calcado; es decir, simplemente facilitar el contenido del libro (Cordero y Svarzman, 2007).

El apego a esta formación educativa sencilla, fácil y acomodaticia, se ha convertido en una contribución para desnaturalizar el fomento innovador de la Educación Geográfica, en el desarrollo curricular de la geografía en la escuela. Precisamente, la didáctica empleada es considerablemente reduccionista, pues tan solo se dedica a ejercitar la transmisión de contenidos programáticos, sin su aplicabilidad para estudiar temas y problemas ambientales, geográficos y 
sociales. Esa tradición implica que la tarea formativa es tan solo una contribución para educar intelectuales enciclopedistas desfasados de su territorio.

De allí que sea razonable que la Educación Geográfica debería apoyar la alfabetización de esta disciplina, de tal manera que tanto el docente como sus estudiantes, superen la condición de espectador pasivo, acrítico e indiferente. Ante las circunstancias del mundo contemporáneo, su labor educativa debería contribuir a formar a los ciudadanos como seres humanos de criterio autónomo, crítico y creativo. Un aspecto a desarrollar, en consecuencia, es superar la reproducción del contenido programático por el ejercicio pedagógico cotidiano de la elaboración del conocimiento. Eso supone aplicar la investigación social que facilite la comprensión de la realidad geográfica comunitaria.

El reto que surge para la enseñanza de la geografía, es acercar al estudiante a su realidad espacial real y concreta, mediante propuestas metodológicas que no sólo le permitan categorizar desde el saber científico las características que encuentra en su espacio geográfico específico, es necesario que en la enseñanza de geografía se recurra también a los saberes que se encuentran fuera de la escuela, como un complemento o como un factor esencial en los currículos y en las propuestas didácticas para el aprendizaje de la geografía y de su esencia de estudio: el espacio social (Cabaluz, 2015, p. 6).

Se trata de asumir los sucesos ocurridos en el escenario próximo a la geografía; en especial, las dificultades confrontadas por los habitantes de la localidad, con acciones indagatorias facilitadoras de la obtención de puntos de vista de los ciudadanos, ante el interrogatorio formulado. Esta actividad tendrá como efecto significativo revelar la opinión de la colectividad sobre un determinado problema comunitario (Sastoque y Ávila, 2016). Así, en los procesos de enseñanza y de aprendizaje, se podrá realizar el desciframiento de los datos obtenidos, con efecto sensibilizador de la conciencia crítica y constructiva, como oportunidad de fomentar el compromiso y la responsabilidad social de un territorio humanizado. Es reivindicar la experiencia personal y el protagonismo social en la explicación de la realidad vivida.

De esta forma se realiza la apertura a los escenarios donde se desenvuelve la dinámica de la sociedad, en sus complejidades, incertidumbres y contrasentidos, como también facilita estimular el viraje epistémico de la formación del ciudadano espectador que contempla pasivamente las 
situaciones, por un ciudadano analítico, cuestionador, creativo y participante, involucrado activamente en la explicación crítica de los cambios del entorno. La importancia pedagógica de este cambio epistémico representa para la geografía escolar, asumir los fundamentos de la Educación Geográfica, en lo relacionado con educar para entender las necesidades de la comunidad y la transformación social.

Entre los desafíos a asumir en la geografía escolar, está en primer lugar, superar el determinismo geográfico, tendencia de mayor tradición en nuestro país, la cual se ofrece desde los textos escolares, en los que se incluyen metodologías inadecuadas como la de copiar y transcribir textos a partir de preguntas y respuestas, realizar localizaciones de lugares o enumerar accidentes físico-naturales y las divisiones continentales, sin argumentación alguna que implique comprensión de lo que se dice (Martínez y Quiroz, 2012, p. 4).

Como la finalidad es dar el signo humanista al acto educante, el cambio en los procesos de enseñanza y de aprendizaje es ejercitar los análisis y las reflexiones como base para gestionar la innovación a la influencia transmisiva de la geografía escolar. Es educar en el escenario de los acontecimientos, en el desenvolvimiento de un proceso formativo vinculado con la construcción del conocimiento contextualizado; especialmente, asignar significado al diálogo y a la interpretación, con el propósito de activar otras opciones para aprender en forma constructiva, a través del incentivo del razonamiento crítico y ofrecer ideas originales, creativas e innovadoras, forjadoras de la conciencia cuestionadora.

\section{La Educación Geográfica, la geografía escolar y el fomento del pensamiento crítico}

Las circunstancias del mundo contemporáneo revelan un comportamiento novedoso muy diferente a la evolución histórica, cuyos acontecimientos desdibujan desde las particularidades de las comunidades hasta la visión globalizada actual. Se trata de cambios y transformaciones reveladores de lo comunitario hacia la totalidad planetaria. Esta realidad deja entrever el comportamiento geográfico de los sucesos característicos de cada momento, al igual las reflexiones conducentes a manifestar la comprensión de sus realidades. Por tanto, desde el mito, la leyenda, el cuento, la fe, lo objetivo hasta el conocimiento científico, han sido motivo para entender la realidad vivida. 
Indiscutiblemente que el pensamiento crítico no dista mucho de las implicaciones de lo político y lo ideológico, pues tradicionalmente ha sido invisibilizado en el acto pedagógico con el propósito de evitar contribuir a promover la emancipación de la sociedad de los mecanismos controladores impuestas por la política hegemónica y la dominación. Precisamente se evita ejercitar el pensamiento reflexivo pues su afán explicativo, coloca en el primer plano a la casualidad que origina al objeto de estudio, tanto en la perspectiva visual, como en la lectura hermenéutica de lo oculto a lo real. Por tanto, eso supone la posibilidad de interpretar la realidad geográfica en forma integral.

Ahora bien, el pensamiento crítico también es crítico de los sentidos comunes de quienes buscamos enfrentar esas hegemonías. No se trata de equidistancia alguna: el pensamiento crítico toma partido, pero no cree -y vaya si tiene motivos para ello- que de la toma de partido se derive alguna verdad o alguna obviedad indiscutible, alguna religión intocable, alguna palabra que ya lo haya resuelto todo. (Aricó, et al., 2015, p. 12).

Desde esta perspectiva, el incentivo es educar para comprender la influencia nefasta del modelo educativo transmisivo y la obsolescencia de la orientación alfabetizadora de la geografía escolar. Esta problemática incide en revisar la disposición de la práctica escolar de las asignaturas geográficas, en especial, la explicación geográfica positiva pues poco confiable para entender la inestabilidad e incertidumbre de las condiciones históricas globalizantes, cuyas características debilitan su capacidad para explicar los objetivos de las Ciencias Sociales, por ejemplo, la geografía cuya realidad esta alterada en forma cotidiana por sus aligeradas mutaciones.

En esta situación emergen otras exigencias formativas de los ciudadanos e implica impulsar una educación más acertada con la comprensión de los complicados y difíciles acontecimientos vividos, en especial, considerar la formación del ciudadano culto, autónomo, crítico y creativo. Eso supone adecuar los procesos de enseñanza y de aprendizaje orientados con la teoría y la práctica propia y adecuada a su naturaleza epistemológica. De allí el interés en el propósito de interpretar en forma razonada y crítica, la realidad vivida.

En el ámbito educativo, es urgente revisar las acciones pedagógicas y didácticas que se aplican en la geografía escolar. Es indispensable entender que la Educación Geográfica debe estrechar la armonía conceptual y práctica de la geografía, con los avances pedagógicos y didácticos, con el objeto de orientar las acciones que 
gestionan la alfabetización geográfica, a partir del abordaje en forma metódica, razonada, critica y creativa, de las dificultades generadas por la relación entre la sociedad y la naturaleza (Davini, 2015, p. 11).

Una opción reiterativa y muy común desde los años ochenta del siglo XX, hasta el presente, ha sido estimular en la geografía escolar la aplicación de la investigación participante. Por eso urge prestar atención al análisis crítico de las situaciones cotidianas y ofrecer perspectivas de cambio más acordes a las necesidades educativas contemporáneas. Un aporte significativo es utilizar en la renovación de la geografía escolar, los fundamentos de la ciencia cualitativa interpretativa. Así, es factible reflexionar sobre el entorno inmediato y estructurar opciones factibles de promover el cambio social a las dificultades vividas en el marco histórico del mundo contemporáneo.

Un punto de partida para impulsar el cambio en la formación que motiva la Educación Geográfica es desarrollar propuestas pedagógicas donde lo esencial debe ser aportar contribuciones fortalecedoras de agitar la reflexión en el cumplimiento de la actividad didáctica. Eso apunta a superar al pensamiento único y a la memorización, debido a sus consecuencias distorsionadoras en evitar o desviar el aprendizaje fomentador del análisis, la reflexión y el cuestionamiento. Al respecto, se promueve desde el mismo siglo XIX, ejercitar el razonamiento sobre los contenidos programáticos, como de la propia realidad objeto de estudio (Balaguer, 2018).

Es decir, el desafío es activar la enseñanza y el aprendizaje centrado en la resolución de problemas. Eso supone contradecir el acto reproductivo por la construcción del conocimiento como una tarea pedagógica sustentada en la razón activa como sostén en la formación pedagógica crítica. Lo cierto es que, ante la realidad geográfica compleja y citica, la racionalidad cuestionadora debe ser cotidiana en la labor formativa de aula de clase de la geografía escolar. En este sentido, la práctica escolar cotidiana afecta a la memorización, resulta contradictoria ante el planteamiento innovador del pensamiento crítico, pues en la geografía en la escuela, se debe motivar el diálogo, el intercambio de ideas, la controversia intencionada y la apertura del debate sobre temas y problemas geográficos.

En otro plano y recogiendo algunos de los planteamientos de la Geografía Crítica desarrollada en América Latina, una pedagogía territorializadora no sólo implica el tomar los elementos presentes en el espacio donde se está, sino que debe proponer 
como eje estratégico la re-apropiación (material y simbólica) del espacio, construyendo relaciones sociales coherentes con las necesidades, intereses y proyecciones de las comunidades y los sujetos que la componen, configurando nuevas identidades y nuevos lazos de afectividad con el espacio y entre sí (Cabaluz, 2015, pág. 43).

En la generalidad de los casos, precisamente un punto estimulante es debatir las situaciones habituales de la comunidad, con el propósito de obtener planteamientos que faciliten comprender las razones explicativas del objeto de estudio. La base de la discusión es observar un problema y someter al análisis en forma abierta y constructiva. Lo esencial será comprender críticamente lo analizado. Igualmente, otro aspecto que potencia el pensamiento crítico es, por ejemplo, analizar e interpretar un texto escrito sobre una temática geográfica. Eso significa leer juiciosamente la realidad geográfica, para luego debatir lo leído en grupos de trabajo y, a continuación, juzgar en forma crítica y constructiva el objeto de estudio.

Es imprescindible facilitar la práctica pedagógica con el acento esencial y básico en el fomento de la conversación reveladora de constructos originados en la actividad del pensamiento en lo que respecta a los procesos reflexivos; en especial, derivados de la labor derivada de la conversación argumentada en el aula de clase. Se trata de la exposición explicativa de puntos de vista estructurados en el ejercicio del pensar en la dirección analítica constructiva. Desde ese accionar se estará en capacidad de construir una subjetividad originada en la participación y el protagonismo social, al realizar la lectura constructiva del pensamiento crítico (García de la Vega, 2002).

\section{Consideraciones finales}

El desenvolvimiento del mundo contemporáneo, a partir de los años ochenta del siglo XX, se pueden considerar como el punto de partida de una racionalidad de acento crítico en la enseñanza de la geografía, con el propósito de replantear la acción formativa de los ciudadanos, al proponer la innovación de la memorización acostumbrada como la manifestación clásica del aprendizaje, a potenciar en las asignaturas geográficas. El motivo reiterativo ha sido reproducir, trascribir y copiar el contenido geográfico a aprender de la manera más sencilla de acceder a preservarlo, tal y como aparece en el libro, por ejemplo. 
Implica, ante los cambios y transformaciones reveladas en las condiciones de la época, la Educación Geográfica, ante sus renovados e innovadores planteamientos teóricos para mejorar su calidad educativa, curricular y pedagógica. Sin embargo, cuando se prestó atención a la práctica escolar cotidiana con los conocimientos y actividades del enfoque cualitativo de la ciencia, se formuló el cuestionamiento a la inmutabilidad e infalibilidad aseguradas en los planificadores de los currículos, en lo referido a la impresionante estabilidad de lo conceptual, lo epistemológico y lo educativo decimonónico, en la enseñanza de la geografía.

La misión innovadora apuntó hacia considerar que la vigencia de la ciencia positivista garantizaría la calidad científica y pedagógica, como educar los ciudadanos con una labor garante de facilitar una opción coherente, pues permite comprender los acontecimientos del mundo globalizado complejo y caótico, con una orientación educativa de fortaleza científica y pedagógica. Sin embargo, en los años finales del siglo XX, los objetos de estudio en las Ciencias Sociales requirieron ante nuevas circunstancias, el requerimiento de otras explicaciones, ahora más relacionados con los testimonios críticos de los actores de los problemas sociales.

De esta realidad emergió en los planeamientos de la Educación Geográfica, la necesidad de comenzar por entender que la enseñanza no debería continuar con una práctica geográfica limitada a entender los aspectos físico-naturales del territorio y menos memorizar los detalles geográficos que le caracterizan. Por el contrario, se recomienda el fomento de respuestas pertinentes a los cambios cotidianos, al igual a las transformaciones de la época. En efecto, se trata de renovar el acto educante centrado en facilitar otras explicaciones a la realidad geográfica para entender sus sucesos, no solo al contemplar su existencia, sino también asumir la opción interpretativa de lo real.

Por eso es razonable comprender que al desarrollar nuevas formas de enseñar y de aprender, se impone articular los propósitos de la Educación Geográfica, la geografía escolar y el pensamiento crítico, con la intención de facilitar la explicación científica de los acontecimientos geográficos cotidianos, con la posibilidad de contribuir a vigorizar la conciencia crítica, la responsabilidad y el compromiso social del ciudadano. Indiscutiblemente esta acción educativa implica educar para apoyar la construcción del conocimiento.

Desde esta perspectiva, al proponer la integración entre la escuela, implica relacionar con las condiciones de la época y la geografía escolar. El desafío ya no es transmitir contenidos 176 
programáticos, sino facilitar la actividad didáctica en la resolución de problemas, en la iniciativa por elaborar el conocimiento. En la realidad de la época actual, evidentemente resulta inconveniente centrar la formación en la retención memorística de nociones y conceptos. El requerimiento es ejercitar el desciframiento de la compleja realidad geográfica del mundo globalizado, pues desde el capital se pretende obnubilar los constructos del pensamiento neoliberal al intervenir en forma irracional las potencialidades del territorio.

\section{Referencias bibliográficas}

Aiello, M. (2005). Las prácticas de la enseñanza como objeto de estudio. Una propuesta de abordaje en la formación Docente. Revista EDUCERE, 9(30), 329-332. https://www.redalyc.org/articulo.oa?id=35603008

Aricó, J., Portantiero, J., Nun J., Laclau, E., Donghi, T., Romero, J., Argumedo, A., Torre, J., Lobato, M., García Canclini, N., Kusch, R., Sarlo, B., Schmucler, H., Barrancos, D., Ferrer, A., Schvarzer, J., Jelin, E., Jauretche, A., González, H., y O’Donnel, G. (2015). Antología del pensamiento crítico argentino contemporáneo. Buenos Aires, Argentina: CLACSO.

Ander Egg, E. (2004). Globalización. El proceso en el que estamos metidos. Córdoba, Argentina: Editorial Brujas.

Balaguer Mora, P. (2018). Geografía crítica y pensamiento crítico. Actualidades Pedagógicas, 1(72), 73-95. https://doi.org/10.19052/ap.5232

Cabaluz, F. (2015). Entramando pedagogías críticas latinoamericanas. Santiago de Chile: Quimantú.

Casado, E., y Calonge, S. (2001). Conocimiento social y sentido común. Caracas, Venezuela: Fondo Editorial de La Facultad de Humanidades y Educación, Universidad Central de Venezuela.

Casas Vilalta, M., y Botella Corral, J. (2003). La democracia y sus retos en el siglo XXI.

Elementos para la formación democrática de los jóvenes. Barcelona, España: Praxis.

Cordero, S., y Svarzman, J. (2007). Hacer geografía en la escuela. Buenos Aires, Argentina: Ediciones Novedades Educativas.

Davini, M. C. (2015). La formación en la práctica docente. Buenos Aires, Argentina: Paidós. 
Gallego Badillo, R., y Pérez Miranda, R. (2003). El problema del cambio en las concepciones epistemológicas, pedagógicas y didácticas. Bogotá, Colombia: Universidad Pedagógica Nacional.

García de la Vega, A. (218). Aplicación didáctica del aprendizaje basado en problemas al análisis geográfico. Revista de Didácticas Específicas, (2), 45-19. https://revistas.uam.es/didacticasespecificas/article/view/9201

García Martínez, J. (10 de marzo 2007). Ciencia en el tiempo de la posverdad. Diario El Mundo. https://bit.ly/3touyhg

García Ríos, D. La construcción de pensamiento crítico en el aula de geografía escolar. GeoGraphos. 10(118), 179-205. https://doi.org/10.14198/GEOGRA2019.10.118

García Lastra, M. (2013). Educar en la sociedad contemporánea. Hacia un nuevo escenario educativo. Convergencia, Revista de Ciencias Sociales, 20(62), 199-220.

Maldonado, C. E. (2016). Complejidad de las ciencias sociales. Y de otras ciencias y disciplinas. Bogotá, Colombia: Ediciones desde abajo. https://doi.org/10.4000/artelogie.1609

Martínez Zapata, I. A., y Quiroz Posada, R. E. (2012). ¿Otra manera de enseñar las Ciencias Sociales? Tiempo de Educar, 13(25), 85-109.

Pérez Jiménez, C. (2003). Formación de docentes para la construcción de saberes sociales. Revista Iberoamericana de educación, (33), 37-54. https://doi.org/10.35362/rie330908

Pipkin, D., Varela, C., y Zenobi, V. (2001). Aportes para el debate curricular. Enseñanza de las Ciencias Sociales. Buenos Aires, Argentina: Secretaria de Educación. Dirección de Currícula, Gobierno de la Ciudad Autónoma de Buenos Aires.

Salgado Labra, V. (25 al 29 de julio del 2011). Construyendo prácticas pedagógicas críticas para la enseñanza de la geografía. Ponencia presentada en el XIII Encuentro de Geógrafos de América Latina. Universidad de Costa Rica. Revista Geográfica de América Central, (Número Especial EGAL, 2011- Costa Rica), 1-12.

Sastoque Gutiérrez, D. M., Ávila Palet, J.E, y Olivares Olivares, S. L. (2016). Aprendizaje Basado en Problemas. Voces y Silencios: Revista Latinoamericana de Educación, 7(1), 148-172.

Santiago Rivera, J. A. (2017). La alfabetización geográfica comunitaria desde la práctica escolar cotidiana de la geografía escolar. Revista Brasileira de Educação em Geografia, 7(14), 24-43. https://doi.org/10.46789/edugeo.v7i14.528

Santiago Rivera, J. A. (2013). La Educación Geográfica y el cambio pedagógico de su trabajo escolar cotidiano. Entorno Geográfico, (9), 8-27. https://doi.org/10.25100/eg.v0i9.3631 
Santos, M. (2004). Por otra globalización. Del pensamiento único a la conciencia universal. Bogotá, Colombia: Edición del Convenio Andrés Bello.

Soares, P. R. R., y Ueda, V. (2002). Anotaciones para pensar la enseñanza de la geografía ante los retos de la posmodernidad. Educación y Pedagogía, (34), 87-96. 